\title{
Thermoelastic Formulation of a Hypersonic Vehicle Control Surface for Control-Oriented Simulation
}

\author{
Nathan J. Falkiewicz* and Carlos E. S. Cesnik ${ }^{\dagger}$ \\ Department of Aerospace Engineering, University of Michigan, Ann Arbor, MI 48109, USA \\ Michael A. Bolender ${ }^{\ddagger}$ and David B. Doman ${ }^{\S}$ \\ Air Force Research Laboratory, Wright-Patterson AFB, OH 45433, USA
}

\begin{abstract}
Hypersonic vehicle simulation is complicated due to coupling between aerodynamics, the propulsion system, heat transfer due to aerodynamic heating, and structural deformations. The high speed involved in hypersonic flight results in the existence of a large heat flux at the vehicle surface. Heat transfer through the internal structure leads to temperature gradients that load the structure due to differential thermal expansion. Additionally, the structure is loaded by aerodynamic pressure. Of particular interest in this study is the extent to which structural deformation alters aerodynamic performance as it relates to vehicle control. As the control surfaces are expected to produce a significant portion of the aerodynamic forces and moments on the vehicle, this paper will focus on the integrated aerothermoelastic modeling of such a structure with the goal of evaluating the effect of structural elasticity on flight dynamics and control system performance. The Eckert reference temperature method is used to calculate the aerodynamic heat flux boundary condition at the surface and finite element representations are used to calculate the temperature distribution and structural displacements. The aerodynamic pressures over the deformed configuration are found using the analytical oblique shock/expansion fan relations. The resulting pressures are integrated over the surfaces to calculate the total lift and drag. For the trajectory points considered, the maximum relative change in lift and drag were found to be $2 \%$ and $6 \%$, respectively. A lift-based trim routine is outlined for use in assessing the control surface deflections required to account for the change in aerodynamic lift and drag.
\end{abstract}

\section{Introduction}

Recent research into air-breathing hypersonic vehicles (HSVs) has been aimed at efficiently modeling the the aerodynamics, propulsion system, structural dynamics, and heat transfer and integrating these models into a comprehensive simulation that can be used for stability and controllability analysis and control law design. There are two approaches to developing these models. The first is the development of first-principles models that are low-order, but lack the fidelity found in more complex representations. The second approach is the development of reduced-order models derived from state-of-the-art, high fidelity computational tools. These tools are not utilized for design due to their complexity and long run-times. However, they are useful for generating reduced-order models that capture the essential physics with a relatively low number of states and therefore they can be used for design. The primary difficulty associated with design of HSVs stems from the high degree of coupling they exhibit between the aerodynamics, heat transfer, elastic airframe, propulsion system, flight dynamics, and controls. Due to the strongly coupled nature of hypersonic vehicle design,

\footnotetext{
${ }^{*}$ Graduate Student Research Assistant, Dept. of Aerospace Engineering, 1320 Beal Ave, Ann Arbor, MI 48109, Student Member AIAA.

${ }^{\dagger}$ Professor of Aerospace Engineering, Dept. of Aerospace Engineering, University of Michigan, 1320 Beal Ave, Ann Arbor, MI 48109, Associate Fellow AIAA.

${ }^{\ddagger}$ Aerospace Engineer, AFRL/RBCA, 2210 Eighth St, Bldg. 146/Room 300, Wright-Patterson AFB, OH 45433, Senior Member AIAA.

§Senior Aerospace Engineer, AFRL/RBCA, 2210 Eighth St, Bldg. 146/Room 300, Wright-Patterson AFB, OH 45433 , Associate Fellow AIAA.
} 
multidisciplinary computational tools are needed to address hypersonic vehicle control law performance early in the design stage.

Though HSVs typically consist of a lifting body design to accomodate the propulsion system, the control surfaces are expected to provide a significant contribution to the aerodynamic lift, drag, and moments acting on the vehicle. This leads to the necessity to conduct a thorough analysis of the major interactions between the control surfaces and other vehicle components in order to accurately predict vehicle performance. Due to the high speed involved in hypersonic flight, transient aerodynamic heating will occur at the outer mold line of the control surfaces and heat will be conducted through their internal structure. Due to the resulting spatial variation of temperature throughout the structure, loads will act on the structure due to differential thermal expansion. Additionally, aerodynamic pressure loads will act on the outer surface. Under this combined loading, the structure will displace relative to its initial configuration. This quasistatic deformation of the control surface will result in a modification of the aerodynamic profile of the airfoil, resulting in altered aerodynamic performance. Because the temperature distribution and aerodynamic pressures are changing over the flight trajectory, the aerodynamic performance of the control surface will be changing throughout flight. To characterize the forces and moments acting on the vehicle, it is necessary to characterize how the aerodynamic performance of the control surfaces change over a trajectory. One must therefore perform integrated aerothermoelastic simulations to assess the effect of control surface deformation on its aerodynamics, and ultimately on the overall vehicle flight dynamics and controllability.

Recent studies have been conducted to assess control system performance and vehicle controllability under effects from the disciplines mentioned above. Studies have been carried out to investigate the steady aerodynamics of hypersonic vehicle control surfaces. ${ }^{1,2}$ Oppenheimer et al. ${ }^{1}$ made use of an analytical shock/expansion formulation to calculate aerodynamic flow parameters and assess the interactions between a foreward canard and an aft elevon control surface. This study sought to quantify the effect of the canard on the effectiveness of the elevon. One aspect of the work involved characterizing the slipline at the trailing edge of the canard due to a difference in pressure between the flow coming off of the top and bottom surfaces. Once the properties of the flow impacting the elevon were characterized, a study of the effectiveness of the elevon was conducted. Results showed that the slipline at the trailing edge of the canard can lead to a decrease in the elevon effectiveness. In a related study, Skujins et al. ${ }^{2}$ conducted computational fluid dynamics (CFD) studies to quantify the level of interaction between the canard and elevon for a similar vehicle geometry. Parametric studies were conducted to assess the range of validity of the analytical shock and expansion fan formulation. Following these studies, an ensemble of CFD cases were generated to assess how the elevon effectiveness was affected by the canard. Results showed that the slipline coming off of the trailing edge of the canard does have an impact on the control effectiveness of the elevon. The effect of the slipline on the elevon was found to diminish as the separation between the two airfoils increased. While these studies compared the accuracy of a shock/expansion approach to CFD solutions, the analyses were two-dimensional. Additionally, the control surfaces were taken to be rigid and the pressures over the surfaces did not contain spatial variation resulting from flexibility effects. By permitting the structure to deform under pressure and thermal loads, a potentially significant change in the aerodynamic performance may result.

Piston theory has been a common method applied to capture unsteady aerodynamic effects in hypersonic flow. ${ }^{3-5}$ Oppenheimer and Doman ${ }^{3}$ used first-order piston theory to calculate the aerodynamic forces and moments for a hypersonic vehicle with consideration of unsteady effects due to perturbations in linear velocities and angular rates resulting from rigid body motion. The total forces and moments on the vehicle were then calculated and stability derivatives were found. Flow over the body surfaces and elevator control surfaces hinged at their mid-chord were considered. Initial simulation results showed that the unsteady aerodynamic components will have an impact on the total forces and moments acting on the vehicle. Although this study was a step toward assessing the impact of aerodynamic forces on rigid body vehicle dynamics, neither flexibility nor heating effects were considered.

In a related work, ${ }^{4}$ piston theory was used to calculate the surface pressure distributions, but in this case flexibility effects were also considered. Also in this study, a canard control surface was considered in addition to the elevator surface addressed in Ref. 3. Control surface effects were considered by modeling the velocity and angular rate perturbations on them resulting from fuselage flexibility. While the unsteady aerodynamic effects were shown to have an effect on the poles and zeros of the linearized dynamics, this study did not include the effects of flexible control surfaces. Additionally, thermal effects were not present in this study. In a similar study, Bolender, Oppenheimer, and Doman ${ }^{5}$ added viscous effects to the aerodynamic model and showed that the resulting forces and moments can have a significant impact on the drag and pitching 
moment of the vehicle. Results also showed that the viscous effects were more pronounced than the unsteady effects.

With regard to aerothermal modeling, Culler, Williams, and Bolender ${ }^{6}$ considered the effect of aerodynamic heating on the mode shapes and natural frequencies of a free-free beam representative of an HSV structure. The aerodynamic heat flux at the surface was found using the Eckert reference temperature method for turbulent, high speed flow over a flat plate. A finite difference method was used to calculate the nodal temperatures through the thickness of the structure. In this work, dynamics were considered by using an analytical formulation of the free-free beam equations solved using the assumed modes method. The authors investigated both the effect of mass change due to propellant burn and material stiffness degradation due to aerodynamic heating on the structural dynamics with the overall goal of assessing vehicle dynamics and control. Results showed that the effect of aerodynamic heating largely offset the effect of mass change due to propellant burn, resulting in insignificant changes to the first mode throughout hypersonic cruise. The effects of aerodynamic heating on the rigid-body poles and zeros were found to be negligible, but the real transmission zeros associated with the flight path angle dynamics were found to move to the right. While this study was a step toward aerothermoelastic modeling of an HSV for control considerations, the effects of aerothermal heating on the control surfaces were not considered. In contrast to the analytical beam-like formulation, a higher fidelity, three-dimensional approach such as finite element modeling can more accurately capture the spatial distribution of temperatures and displacements of the control surface structure.

A more recent effort ${ }^{7}$ provided further insight into the coupling between aerodynamic heating and structural deformation through an aeroelastic panel study. In this work, a two-dimensional flexible panel located on the upper surface of a rigid wedge-shaped forebody was considered. Third-order piston theory was used to calculate the local flow properties for the panel. The aerodynamic heat flux was calculated using Eckert's reference enthalpy method with flow properties evaluated at a reference condition to account for compressibility effects. Fully turbulent flow over the length of the panel was assumed with transition taken to occur one meter upstream of its leading edge. A thermal structure was assumed to consist of an outer radiation shield, a middle thermal insulation layer, and an internal plate structure. Heat transfer was considered to occur in two directions (chord-wise and through-thickness) and was calculated using a finite difference approach. For the thermal boundary conditions, aerodynamic heat flux and radiation to the environment were included on the outer surface, and the interior surface and edges of the panel was taken to be adiabatic. To calculate the structural deformation, a semi-infinite panel in cylindrical bending with simple supports was assumed. An analytical approach was used to solve the equations of motion for a von Karman plate undergoing large displacements. To solve the fully coupled aerothermoelastic problem, a partitioned approach was proposed in which different size time steps were taken in the aerothermal and aeroelastic solutions. Results showed that material property degradation with temperature and the effect of elastic deformation on aerodynamic heating were two of the most significant factors influencing the flutter boundary of the panel. Another important result of that work was that inertial effects on the aerothermoelastic response were found to be minimal, thus permitting a quasi-static approach, as will be employed in this work. Although Ref. 7 successfully implemented an overall framework for coupled aerothermoelastic analysis of a panel, greater fidelity is needed to more thoroughly assess the impact of aerothermoelastic effects on HSV control surface dynamics. As opposed to an analytical solution of structural dynamics equations of motion, the numerical approach employed here will capture three-dimensional effects. Additionally, we will integrate the aerodynamic pressures over the surface of the structure and analyze the effect of the changing aerodynamic forces on vehicle controllability.

A numerical approach was taken to solve the thermoelastic problem as applied to a structural model representative of a hypersonic vehicle control surface in another recent work. ${ }^{8}$ The goal of that work was to develop a reduced order modeling (ROM) framework for calculating the natural frequencies and mode shapes of the structure as a function of thermal state due to coupling through material property degradation with temperature. Motivation to consider ROMs resulted from the fact that control-oriented models require formulations that are computationally efficient and possess a low number of states. A modal approach was proposed for the solution of both the thermal and structural dynamics problems, with Proper Orthogonal Decomposition (POD) modes being used in the thermal problem and reference free vibration modes for the structural dynamics. Results for the thermal problem showed good agreement between nodal temperatures from the POD-based ROM and high-fidelity finite element analysis (FEA). A modified modal formulation for the structural dynamics equations of motion was described in which the modal matrix was assembled using the free vibration modes at a reference thermal state representing an average temperature distribution. 
In this formulation, the modal matrix remains constant throughout the analysis, but the stiffness matrix is updated at every time step based on a functional representation of Young's Modulus with temperature. An initial assessment of the overall thermoelastic solution process demonstrated coupling in the structural equations of motion due to the loss of orthogonality with respect to stiffness resulting from the stiffness matrix changing with temperature. While this work provided a reduced order numerical approach for capturing the thermoelastic response of HSV structures, the surface heat flux was assumed and not calculated based on flow properties. The applied heat flux did not therefore represent the spatial distribution that would exist on an actual structure, and did not possess the transient nature seen in hypersonic flight. Additionally, the free vibration problem was considered, but the forced vibration problem was not. Thus, the influence of aerodynamic and thermal loads on the structural response was not taken into account. Furthermore, the analysis did not include an aerodynamic component. Thus, the effect of structural deformation on control surface aerodynamics was not considered.

The progress and limitations described above have led to the creation of a framework for aerothermoelastic analysis of HSV control surfaces for the purpose of assessing overall vehicle flight dynamics and controllability. A major objective of this work is to provide fundamental characterization of the impact of structural deformation due to aerodynamic and thermal loads on aerodynamic forces and moments and to assess how they affect vehicle stability. The salient features of this work include:

- An aeroheating model to calculate nodal convective heating values at the outer surfaces of the control surface.

- A thermal finite element representation of the structure to solve the transient heat transfer problem in order to compute the temperatures within the structure.

- A structural finite element representation to find the nodal displacements resulting from aerodynamic and thermal loads.

- An aerodynamic model to determine the spatially varying flow parameters over the deformed structure.

- A trim routine to adjust the angle-of-attack of control surface to produce the commanded forces and moments.

A flowchart showing all of the major analysis components is shown in Fig. 1. Note that at this time the gray blocks indicating feedback from the aerodynamic flow parameter calculations to the the structural and thermal models have not been implemented, but are currently being addressed.

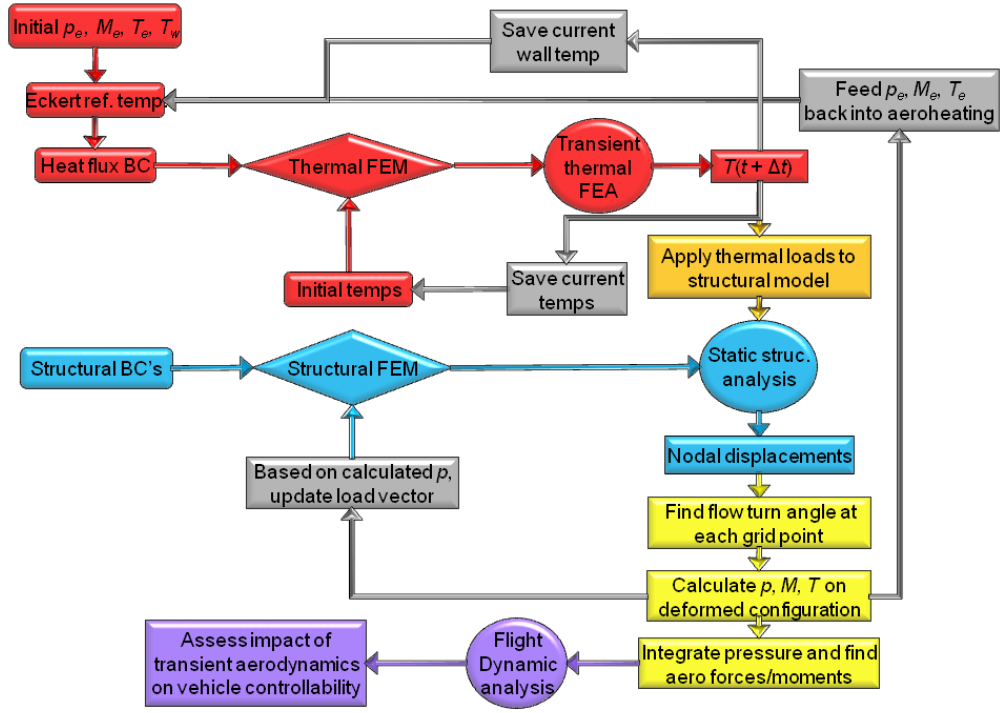

Figure 1. Aerothermoelastic simulation framework to be used in control surface study.

For the aeroheating analysis, Eckert's reference temperature method along with the Schultz-Grunow relation is proposed for calculating the heat transfer coefficient between the boundary layer and the outer 
surface. To determine the temperature distribution through the structure, a thermal finite element model is used with heat flux boundary conditions from the aeroheating model and radiation to the environment. A load case based on the temperatures and aerodynamic pressures is created and a linear static finite element structural solution is used to calculate the nodal displacements. These displacements are then used in an aerodynamic solver that uses the analytical shock/expansion equations ${ }^{1,2}$ to calculate the local aerodynamic pressures at the nodes. The pressures are integrated to find the aerodynamic forces and moments on the surface. A trim routine is then used to adjust the angle-of-attack of the control surface to produce the desired lift. The process is repeated at successive time instants along a trajectory and inertial effects are neglected so as to allow for a quasi-static analysis at each trajectory point. Another goal of this study is to assess the contribution of aerodynamic loads and thermal loads to the structural deformation. Independent analysis of the displacements due to aerodynamic pressure loads and displacements due to thermal loads will permit analysis of the contribution of each to the aerothermoelastic problem and will guide future studies. Details of the formulations used in the aeroheating, heat transfer, structural, aerodynamics, and control models are given in the subsequent sections.

\section{Control Surface Model}

Motivation to consider the thermoelastic response of hypersonic vehicle control surfaces stems from previous development of a two-dimensional hypersonic vehicle configuration. ${ }^{1-5}$ The body of the configuration under consideration in this study consists of a forebody compression ramp, an engine/nacelle segment, an aftbody expansion segment, and a flat upper surface. As shown in Fig. 2, the vehicle is equipped with an elevator control surface. The control surface geometry to be used in this study is based on that from a previous study, ${ }^{8}$ and is given in Fig. 3 .

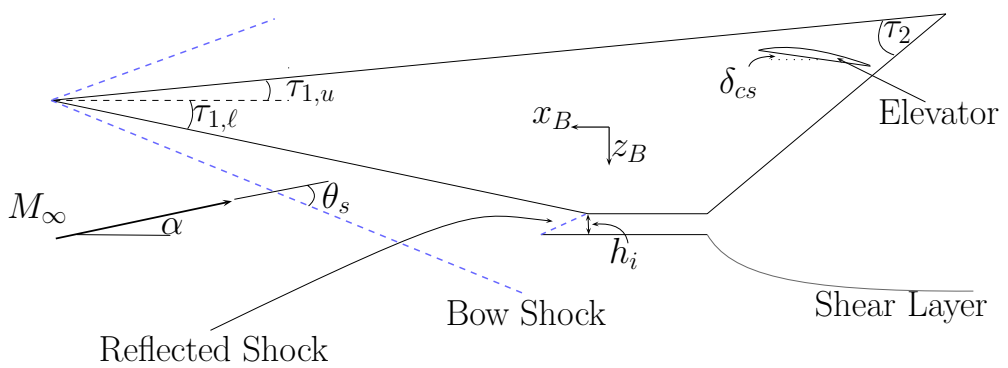

Figure 2. Overall HSV geometry illustrating position of control surface.

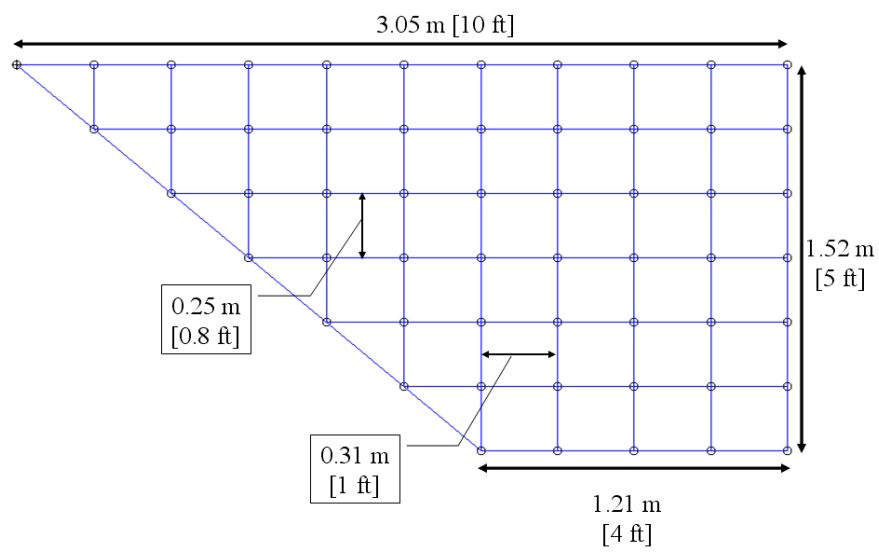

Figure 3. Control surface geometry and dimensions. 
The structure is taken to be made of Ti-6Al-2Sn-4Zr-2Mo due to its high melting temperature and strength-to-weight ratio. The thermal and structural material properties as used in this study are given in Table 1. Based on this geometry, a finite element model of the control surface has been created for the thermal and structural analyses. The model consists of a top and bottom skin surface and is stiffened by internal stiffeners as well as stiffeners around the outer perimeter according to the dimensions specified in Fig. 3. The finite element model is shown in Fig. 4 with the top skin removed for visualization purposes. The model is composed of 9,200 three-node triangular elements all having a thickness of $12.7 \mathrm{~mm}$ (0.5 in). The red circle indicates the area containing displacement constraints in all six degrees of freedom.

Table 1. Thermal and structural material properties for Ti-6Al-2Sn-4Zr-2Mo used in the study. ${ }^{9}$

\begin{tabular}{l|r}
\hline \hline Thermal Conductivity & $6.89 \mathrm{~W} / \mathrm{m} / \mathrm{K}$ \\
\hline Specific heat & $463 \mathrm{~J} / \mathrm{kg} / \mathrm{K}$ \\
\hline Density & $4540 \mathrm{~kg} / \mathrm{m}^{3}$ \\
\hline Poisson's Ratio & 0.32 \\
\hline Young's Modulus & $113.8 \mathrm{GPa}$ \\
\hline Coefficient of Thermal Expansion & $8.1 \mu \mathrm{m} / \mathrm{m} / \mathrm{K}$ \\
\hline \hline
\end{tabular}

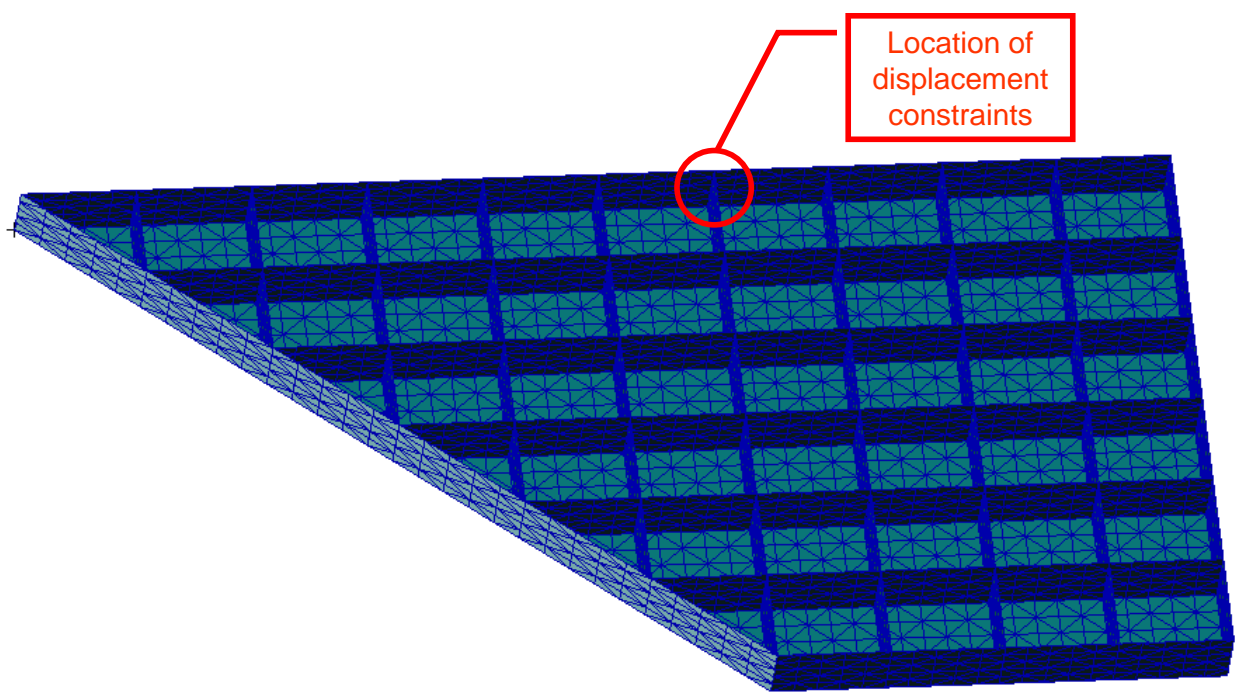

Figure 4. Control surface finite element model.

\section{Aerothermal Modeling}

In order to obtain the temperature distribution associated with the thermal load applied to the structure, the transient heat transfer problem must first be solved. Solution of the heat transfer problem requires boundary conditions at the outer surfaces of the model and initial temperature conditions. For the initial condition, the structure is assumed to be at a uniform temperature of $311 \mathrm{~K}\left(100^{\circ} \mathrm{F}\right)$. For the boundary conditions, this study considers a convective heat flux due to aerodynamic heating, and radiaton to the atmosphere at the outer surface. As this analysis considers a cruise trajectory at $26 \mathrm{~km}(85,000 \mathrm{ft})$, the atmospheric temperature is taken to be constant at $222.5 \mathrm{~K}$. Though the structure of the control surface is taken to be a titanium alloy, radiation properties of a material likely to be used as a radiation shield are employed in the calculation of the radiation heat transfer between the surface and the environment. Based on studies of material stacking schemes, ${ }^{10}$ this work assumes that the radiation shield is made of PM 2000. Thus, the emissivity at the outer surface is taken to be 0.7 .

In order to calculate the spatially varying heat flux resulting from aerodynamic heating, the local freestream pressure, temperature, and Mach number must first be found. To calculate the initial values 
of these properties, an aerodynamics solution (to be described in a subsequent section) is generated based on the undeformed configuration of the model. Once the values of these aerodynamic parameters are known at each node of the model, the values from corresponding nodes of each element are averaged and treated as constant over the element so that an element-uniform heat flux can be calculated. At this point, the heat flux is treated as constant and is not updated as the structure deforms and as the flow parameters change. Future work will likely implement the ability to update the heat flux at certain time intervals. From Holman, ${ }^{11}$ the convective heat flux at the surface is given by

$$
\dot{q}_{\text {aero }}=h\left(T_{w}-T_{r}\right),
$$

where $h$ is the heat transfer coefficient, $T_{w}$ is the wall temperature, and $T_{r}$ is the recovery temperature or adiabatic wall temperature. Because the process that brings the fluid to rest in the boundary layer is irreversible, and because not all of the free-stream kinetic energy is converted to thermal energy in this process, ${ }^{11}$ a recovery factor is used, which is given by

$$
r=\frac{T_{r}-T_{e}}{T_{t}-T_{e}}
$$

where $T_{e}$ is the temperature at the outer edge of the boundary layer and $T_{t}$ is the total temperature or stagnation temperature. The total temperature is given by

$$
T_{t}=T_{e}\left[1+(\gamma-1) \frac{M_{e}^{2}}{2}\right],
$$

where $M_{e}$ is the Mach number at the edge of the boundary layer, $T_{e}$ is the temperature at the edge of the boundary layer, and $\gamma$ is the ratio of specific heats which is taken to have the constant value of 1.4 in this study. This study assumes turbulent flow, thus the recovery factor is given by

$$
r=\operatorname{Pr}^{1 / 3},
$$

where the Prandtl number, $\operatorname{Pr}$, is approximated by assuming a constant value of 0.7 in this study. ${ }^{11}$ Future studies are to be conducted to assess the impact of not including the temperature dependence of the Prandtl number. Due to the large property variations across the boundary layer in the hypersonic flow, the constantproperty heat transfer relations are used with the properties evaluated at a reference temperature, $T^{*}$, as proposed by Eckert. ${ }^{12}$

$$
T^{*}=T_{e}+0.50\left(T_{w}-T_{e}\right)+0.22\left(T_{r}-T_{e}\right) .
$$

Once the reference temperature is known, the reference density, $\rho^{*}$, and reference viscosity, $\mu^{*}$, are calculated using the ideal gas law and Sutherland's law, respectively. With these reference quantities known, the Reynolds number is found using

$$
R e_{x}^{*}=\frac{\rho^{*} V_{e} x}{\mu^{*}},
$$

where $x$ is the distance from the leading edge and $V_{e}$ is the velocity of the flow at the outer edge of the boundary layer. With the local Reynolds number known, the skin friction coefficient, $c_{f}^{*}$, is calculated using the Schultz-Grunow relation which accurately represents measurements with Reynolds numbers up to $10^{9}$ and is given by ${ }^{13}$

$$
c_{f}^{*}=\frac{0.370}{\left(\log _{10} R e_{x}^{*}\right)^{2.584}} .
$$

Once the skin friction is known, the reference Stanton number can be found using the Colburn-Reynolds analogy,

$$
S t^{*}=\frac{c_{f}^{*}}{2} \operatorname{Pr}^{-2 / 3},
$$

and the heat transfer coefficient is found using the definition of Stanton number,

$$
h=S t^{*} c_{p} \rho^{*} V_{e} .
$$

Note that the flow is assumed to be calorically perfect for the purposes of this study and thus a constant value of specific heat, $c_{p}$, is used. Using the heat flux and radiation boundary conditions along with the initial temperatures, the temperature distribution is propagated forward in time using a transient thermal solution. 


\section{Heat Transfer Modeling}

Once the thermal boundary conditions and initial conditions are specified, it remains to solve the finite element heat transfer equations for the nodal temperatures. The finite element formulation for the heat transfer problem begins with the governing energy equation for heat transfer in a three-dimensional anisotropic solid $\Omega$ bounded by outer surface $\Gamma$ given by ${ }^{14}$

$$
-\left(\frac{\partial q_{x}}{\partial x}+\frac{\partial q_{y}}{\partial y}+\frac{\partial q_{z}}{\partial z}\right)+Q=\rho_{s} c_{s} \frac{\partial T}{\partial t}
$$

where $q_{x}, q_{y}$, and $q_{z}$ are the components of the heat flow rate vector in cartesian coordinates, $Q$ is the volumetric internal heat generation rate, $\rho_{s}$ is the density of the material, $c_{s}$ is the specific heat, $T$ denotes temperature, and $t$ denotes time. Note that the subscripts, $s$, on the density and specific heat are used to indicate that these are properties of the structure as opposed to the fluid properties for air used previously. For an isotropic medium, the components of the heat flow rate vector are given by

$$
q=-k \nabla T,
$$

where $k$ is the thermal conductivity of the material. Note that the minus sign is needed due to the fact that heat is transferred from warmer regions to cooler regions. Substituting Eq. (11) into Eq. (10) and assuming constant thermal properties and no internal heat generation yields the heat equation given by

$$
\nabla^{2} T=\frac{1}{\alpha_{T}} \frac{\partial T}{\partial t},
$$

where $\alpha_{T}$ is the thermal diffusivity given by

$$
\alpha_{T}=\frac{k}{\rho_{s} c_{s}} .
$$

Now that the governing equations have been given, we will derive the resulting finite element equations for a single element for simplicity. Using the method of weighted residuals with the governing equation, we require

$$
\int_{\Omega^{(e)}}\left(\frac{\partial q_{x}}{\partial x}+\frac{\partial q_{y}}{\partial y}+\frac{\partial q_{z}}{\partial z}+\rho_{s} c_{s} \frac{\partial T}{\partial t}\right) N_{i} d \Omega=0
$$

where $\Omega^{(e)}$ is the domain for element $(e)$ and $N_{i}$ are the element shape functions. Integrating the term

$$
\int_{\Omega^{(e)}}\left(\frac{\partial q_{x}}{\partial x}+\frac{\partial q_{y}}{\partial y}+\frac{\partial q_{z}}{\partial z}\right) N_{i} d \Omega
$$

by Gauss's theorem introduces surface integrals of the heat flow across the element boundary. After this integration Eq. (14) becomes

$$
\int_{\Omega^{(e)}} \rho_{s} c_{s} \frac{\partial T}{\partial t} N_{i} d \Omega-\int_{\Omega^{(e)}}\left[\frac{\partial N_{i}}{\partial x} \frac{\partial N_{i}}{\partial y} \frac{\partial N_{i}}{\partial z}\right]\left\{\begin{array}{c}
q_{x} \\
q_{y} \\
q_{y}
\end{array}\right\} d \Omega=-\int_{\Gamma^{(e)}}(q \cdot \hat{n}) N_{i} d \Gamma, \quad i=1,2, \ldots, r,
$$

where $\hat{n}$ is the unit normal to the surface, $\Gamma^{(e)}$ is the element surface boundary, and $r$ is the number of nodes of the element. We now express the temperatures and temperature gradients in terms of the shape functions and nodal values of temperature using

$$
\begin{aligned}
T^{(e)}(x, y, z, t) & =N(x, y, z) \mathcal{T}(t) \\
\frac{\partial T^{(e)}(x, y, z, t)}{\partial x_{i}} & =B(x, y, z) \mathcal{T}(t),
\end{aligned}
$$

where $B$ is the shape function derivative matrix, $\mathcal{T}$ indicates the vector of nodal temperatures for the element, and $x_{i}$ indicates the three cartesian coordinate directions. After some manipulation, the resulting system of finite element equations becomes

$$
C \frac{\partial T}{\partial t}+K_{c} T=R_{q}+R_{\sigma}
$$

where $C$ is the thermal capacitance matrix, $K_{c}$ is the thermal conductivity matrix, $R_{q}$ is the thermal load vector resulting from the aerodynamic heat flux, and $R_{\sigma}$ is the thermal load vector resulting from surface radiation. 


\section{Structural Modeling}

A finite element formulation is also used for calculation of structural displacements due to aerodynamic pressure loads and thermal loads. The model and constraints to be used in this study are shown in Fig. 4. This work assumes that the structural deformation is a quasi-static process and nodal displacements are calculated by executing a linear static analysis. Temperature change gives rise to only normals strains, and the resulting strains for an isotropic material are given by

$$
\epsilon_{x x, T}=\epsilon_{y y, T}=\epsilon_{z z, T}=\alpha \Delta T, \epsilon_{x y, T}=\epsilon_{y z, T}=\epsilon_{x z, T}=0,
$$

where the subscript $T$ indicates strains due to thermal effects. The stresses can be found from the strains using the constitutive relation given by

$$
\sigma=D\left(\epsilon-\epsilon_{T}\right)
$$

where $D$ is the elasticity tensor and $\epsilon$ is the tensor of total strains. The total potential energy of the structure is given by

$$
\Pi=\frac{1}{2} \int_{A} \epsilon_{e}^{\mathrm{T}} D \epsilon_{e} t d A-\int_{A} u^{\mathrm{T}} f t d A-\int_{L} u^{\mathrm{T}} T_{s} t d l-\sum_{i} u_{i}^{\mathrm{T}} P_{i},
$$

where $\epsilon_{e}$ is the elastic strain, $t$ is the element thickness, $A$ is the element area, $u$ is the vector of displacements, $f$ contains the body forces acting on the structure, $T_{s}$ contains the surface tractions acting on the structure, $L$ is the length of the edge containing the surface tractions, and $P_{i}$ are the point loads acting on the structure. The relationship between elastic strain, $\epsilon_{e}$, thermal strain, $\epsilon_{T}$, and total strain, $\epsilon$, is given by ${ }^{15}$

$$
\epsilon_{e}=\epsilon-\epsilon_{T} .
$$

The first term in Eq. (21) is the strain energy term, $U$, which will give rise to the thermal loads. Including the effect of thermal strain in the expression of potential energy, the strain energy term becomes ${ }^{16}$

$$
\begin{aligned}
U & =\frac{1}{2} \int_{A}\left(\epsilon-\epsilon_{T}\right)^{\mathrm{T}} D\left(\epsilon-\epsilon_{T}\right) t d A \\
& =\frac{1}{2} \int_{A}\left(\epsilon^{\mathrm{T}} D \epsilon-2 \epsilon^{\mathrm{T}} D \epsilon_{T}+\epsilon_{T}^{\mathrm{T}} D \epsilon_{T}\right) t d A,
\end{aligned}
$$

where $t$ and $A$ are the thickness and area of the structure, respectively. Upon minimization of the potential energy, the first term in the above gives the standard element stiffness matrix, the last term disappears, and the middle term yields the element thermal load vector given by ${ }^{16}$

$$
f_{T}^{e}=t_{e} A_{e} B^{\mathrm{T}} D \epsilon_{T}
$$

where $B$ is the shape function derivative matrix. The contribution of load due to pressure, $f_{p}$, is calculated by distributing the element-uniform aerodynamic pressure load over each element into equivalent nodal point forces. Once the full linear static system is assembled, it yields

$$
K q_{d}=f_{T}+f_{p}
$$

where $K$ is the global stiffness matrix assembled from the local element stiffness matrices, $q_{d}$ is the global degree-of-freedom vector containing the nodal displacements, $f_{T}$ is the global thermal load vector, and $f_{p}$ is the global pressure load vector. The system can be readily solved for the nodal displacements, $q$, such that the deformed configuration can be generated. Once the deformed configuration is known at the time instant of interest, it remains to calculate the aerodynamic flow parameters over the structure.

\section{Aerodynamic Modeling}

Though the thermal and structural analyses are based on models containing a finite and uniform airfoil thickness, the aerodynamic analysis in this work will treat the airfoil as if it were a zero-thickness plate and neglect leading edge, trailing edge, and thickness effects. Therefore, though the finite element mesh employed in the thermal and structural analyses is used aerodynamic flow calculations, the top and bottom surfaces of this model are treated as if they compose the top and bottom surfaces of a plate with no thickness for 
aerodynamics purposes. Inherent in this formulation is the assumption that the deformations of the top and bottom surfaces are approximately the same, and that the deformation of one surface with respect to the other (i.e., local change in airfoil thickness due to deformation) is negligible as compared with the overall gross deformation of the airfoil. The use of three-dimensional models for the thermal and structural analyses is required because one of the goals of this study is to quantify the effect of displacements due to thermal expansion. In order to accurately capture the spatial variation of temperature gradients and the resulting displacements due to differential thermal expansion, a three-dimensional model for thermal and structural analyses is required. However, at this point the aerodynamic formulation only considers the top and bottom surfaces of that model and is unable to account fully for three-dimensional aerodynamic effects. Future work is thus warranted to extend the current formulation to include a more representative airfoil profile.

The methodology for calculation of aerodynamic flow properties over the surfaces is based on the analytical oblique shock and Prandtl-Meyer expansion fan formulation applied in previous work. ${ }^{1,2}$ When a flow is turned into itself in hypersonic flow, an oblique shock will be formed. For the case of an oblique shock, the angle that the shock makes with the freestream, $\theta_{s}$, is first found using ${ }^{17}$

$$
\sin ^{6} \theta_{s}+b \sin ^{4} \theta_{s}+c \sin ^{2} \theta_{s}+d=0,
$$

where the coefficients, $b, c$, and $d$, are given by

$$
\begin{aligned}
& b=\frac{-M_{1}^{2}+2}{M_{1}^{2}}-\gamma \sin ^{2} \delta \\
& c=\frac{2 M_{1}^{2}+1}{M_{1}^{4}}+\left[\frac{(\gamma+1)^{2}}{4}+\frac{\gamma-1}{M_{1}^{2}}\right] \sin ^{2} \delta \\
& d=-\frac{\cos ^{2} \delta}{M_{1}^{4}}
\end{aligned}
$$

where $\delta$ is the local flow turning angle, $M_{1}$ is the Mach number upstream of the shock, and $\gamma$ is the ratio of specific heats. The weak shock solution is selected, and with $\theta_{s}$ known the post-shock flow properties are calculated using ${ }^{17}$

$$
\begin{aligned}
\frac{p_{2}}{p_{1}} & =\frac{7 M_{1}^{2} \sin ^{2} \theta_{s}-1}{6} \\
\frac{T_{2}}{T_{1}} & =\frac{\left(7 M_{1}^{2} \sin ^{2} \theta_{s}-1\right)\left(M_{1}^{2} \sin ^{2} \theta_{s}+5\right)}{36 M_{1}^{2} \sin ^{2} \theta_{s}} \\
M_{2}^{2} \sin ^{2}\left(\theta_{s}-\delta\right) & =\frac{M_{1}^{2} \sin ^{2} \theta_{s}+5}{7 M_{1}^{2} \sin ^{2} \theta_{s}-1},
\end{aligned}
$$

where $M_{1}, p_{1}$, and $T_{1}$ are Mach number, static pressure, and temperature upstream of the shock and $M_{2}$, $p_{2}$, and $T_{2}$ are the Mach number, static pressure, and temperature downstream of the shock.

When a flow is turned away itself, a Prandtl-Meyer expansion fan occurs. The calculation of flow properties behind an expansion begins by calculating the Prandtl-Meyer function, $\nu$, based on the Mach number upstream of the expansion, $M_{1}:{ }^{18}$

$$
\nu\left(M_{1}\right)=\sqrt{\frac{\gamma+1}{\gamma-1}} \tan ^{-1} \sqrt{\frac{\gamma-1}{\gamma+1}\left(M_{1}^{2}-1\right)}-\tan ^{-1} \sqrt{M_{1}^{2}-1} .
$$

Using $\nu\left(M_{1}\right)$ and the flow turn angle, $\delta$, the Prandtl-Meyer function based on downstream Mach number, $M_{2}$, is found using

$$
\nu\left(M_{2}\right)=\nu\left(M_{1}\right)+\delta .
$$

Once $\nu\left(M_{2}\right)$ is known, we can again use the expression for the Prandtl-Meyer function given in Eq. (29), except in this case we use $M_{2}$ instead of $M_{1}$ :

$$
\nu\left(M_{2}\right)=\sqrt{\frac{\gamma+1}{\gamma-1}} \tan ^{-1} \sqrt{\frac{\gamma-1}{\gamma+1}\left(M_{2}^{2}-1\right)}-\tan ^{-1} \sqrt{M_{2}^{2}-1} .
$$


This equation is solved numerically for $M_{2}$ to give the Mach number downstream of the expansion. Because an expansion fan is isentropic, the total pressure and total temperature are constant across the expansion and we can use the isentropic flow relations to calculate the remaining flow properties: ${ }^{18}$

$$
\begin{aligned}
& \frac{T_{2}}{T_{1}}=\frac{1+\frac{\gamma-1}{2} M_{1}^{2}}{1+\frac{\gamma-1}{2} M_{2}^{2}} \\
& \frac{p_{2}}{p_{1}}=\left[\frac{1+\frac{\gamma-1}{2} M_{1}^{2}}{1+\frac{\gamma-1}{2} M_{2}^{2}}\right]^{\frac{\gamma}{\gamma-1}} .
\end{aligned}
$$

In this work these equations are applied in a local sense to capture spatial variations in flow properties over the surface due to deformation. The goal of the aerodynamic model in this paper is to capture the effect of flow turning due to structural deformations on the aerodynamic forces and moments. As opposed to performing computationally intensive CFD analysis at every time step, the use of such an analytical formulation for the aerodynamics model is consistent with the control-oriented modeling philosophy that the framework remain numerically tractable and efficient. Thus, such an approach captures the physics of the spatially varying aerodynamic flow parameters while remaining at a low enough order to be used in control-related studies.

The control surface is taken to be at an angle-of-attack, $\alpha$, of $6^{\circ}$ and a yaw angle, $\beta$, of $0^{\circ}$. The aircraft is assumed to be at a Mach 8 cruise condition at an altitude of $85,000 \mathrm{ft}$ (26 km). As mentioned previously, the flow is assumed calorically perfect and a constant specific heat is used. In future work it is likely that this assumption will be relaxed as further refinements are made. Once the freestream flow parameters are initialized, the structural displacements at the time instant of interest are computed and the deformed configuration is generated. To find the aerodynamic flow properties over the deformed surface using the shock/expansion relations, a flow turning angle must be specified. As the structure has already been discretized using a finite element representation, the same discretization will also be used in the aerodynamics solution. The local flow turning angle at each node is thus found by calculating the relative change in angle between the surface tangent at the current node and the surface tangent at the node directly upstream. To allow for such calculation of the turning angle, the flow is assumed to occur in chordwise strips along the surface and the nodes of the finite element model are arranged to lie in rows along the chordwise direction as shown in Fig. 5. The finite element mesh on the top and bottom surfaces shown in Fig. 5 allows for easy

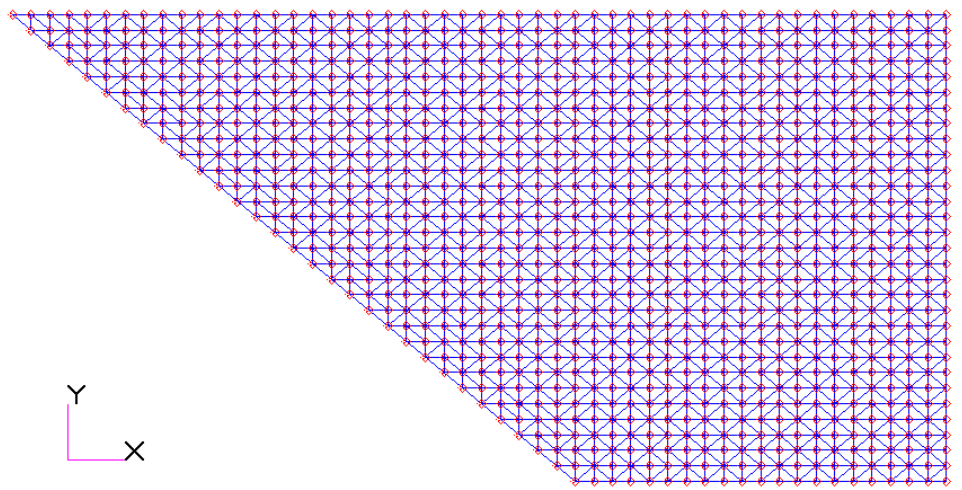

Figure 5. Top surface of finite element model illustrating arrangement of nodes in chordwise rows.

identification of the upstream node. The flow turn angle calculation is done by simply identifying the node at the same station in the y-direction which is the closest node directly upstream. Based on the deformed configuration, the unit vector normal to the surface is found at each node. Note that because a particular node may be connected to multiple elements each having a different normal direction, a standard averaging scheme is employed to best represent the direction normal to the surface at that point. As the flow is taken to occur purely in the chordwise direction and no cross-flow is assumed, the normal is then projected onto a plane parallel to the $\mathrm{x}-\mathrm{z}$ plane. Based on the plane defined by the surface normal, a unit vector tangent to the surface at each node is then found. Again, due to the chordwise flow assumption, we take the tangent that lies in a plane parallel to the x-z plane defined in Fig. 5. 
Once all of the nodal normal and tangent vectors are known, the Mach number, pressure, and temperature can be found at each node. The aerodynamic solution process begins by calculating the flow parameters at the leading edge node at the root of the surface. The flow turning angle is calculated as the angle that the freestream flow vector makes with the surface tangent at this node. Once the flow turning angle is known, the oblique shock or expansion fan relation is used along with the freestream flow properties to calculate the resulting Mach number, pressure, and temperature at that node. The process is successively repeated for each node along the chord in that row of nodes. Once the trailing edge node is reached, the solution moves along the span toward the tip to the next row of nodes until the node at the trailing edge of the tip is reached. It is important to bear in mind that flow parameter calculations for nodes at the leading edge differ from those for nodes in the interior and trailing edge. This is because the interior and trailing edge nodes use the Mach number, pressure, and temperature at the upstream node for the upstream conditions. The leading edge nodes, however, use the freestream properties as the upstream conditions for the flow. Additionally, for nodes not at the leading edge, the flow turning angle is the difference between the angle between the surface tangent at the current node and the surface tangent at the upstream node. As shown in Fig. 6 , the turning angle for node 2 is given by $\varphi_{2}-\varphi_{1}$ and the turning angle for node 3 is given by $\varphi_{3}-\varphi_{2}$.

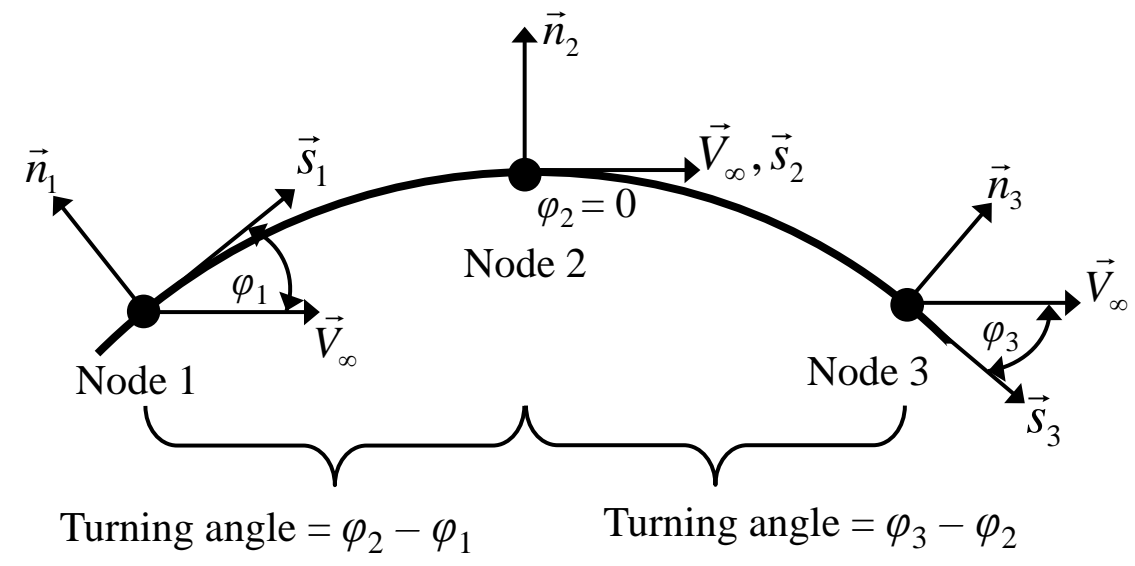

Figure 6. Illustration of calculation of local flow turning angle for use in shock/expansion relations.

With the nodal pressures known at the top and bottom surfaces of the structure, the value of the elementuniform pressure is found by averaging the values at the corresponding nodes for each element. To find the resulting force acting normal to the surface of the element, the elemental pressure value is multiplied by the area of the element. The component of the force contributing to drag is found by projecting the resulting force vector in the direction of the freestream flow. Similarly, the component of the force contributing to lift is found by projecting the force vector in the direction perpendicular to the freestream flow. In addition to pressure forces, the resultant force and moment calculations also include the effect of skin friction. Recall that the skin friction coefficient was already found during heat flux calculations from the Schultz-Grunow relation given by Eq. (7). Using the local skin friction coefficient, the local wall shear stress is given by

$$
\tau_{w}=\frac{1}{2} \rho^{*} V_{e}^{2} c_{f}^{*}
$$

As with the pressure force, the element-uniform wall shear stress is multiplied by the element area to calculate the force acting on element. Because the force due to shear will not necessarily be acting in the direction of the freestream, this force is also resolved into lift and drag components. Once the values contributing to lift and drag from both pressure and shear contributions are found, they are summed to give the overall lift and drag forces on the control surface.

\section{Aerothermoelastic Control Surface Simulation Results}

The integrated aerothermoelastic solution begins by calculating the aerodynamic heat flux over the undeformed structure. As described previously, the nodal flow properties must first be found over this configuration and the heat flux is then calculated using Eckert's reference temperature method. Contour 
plots of the heat flux over the bottom and top surfaces of the undeformed configuration are shown in Fig. 7 . As seen in the figure, the heat flux on the bottom surface is higher in general than that on the top surface due

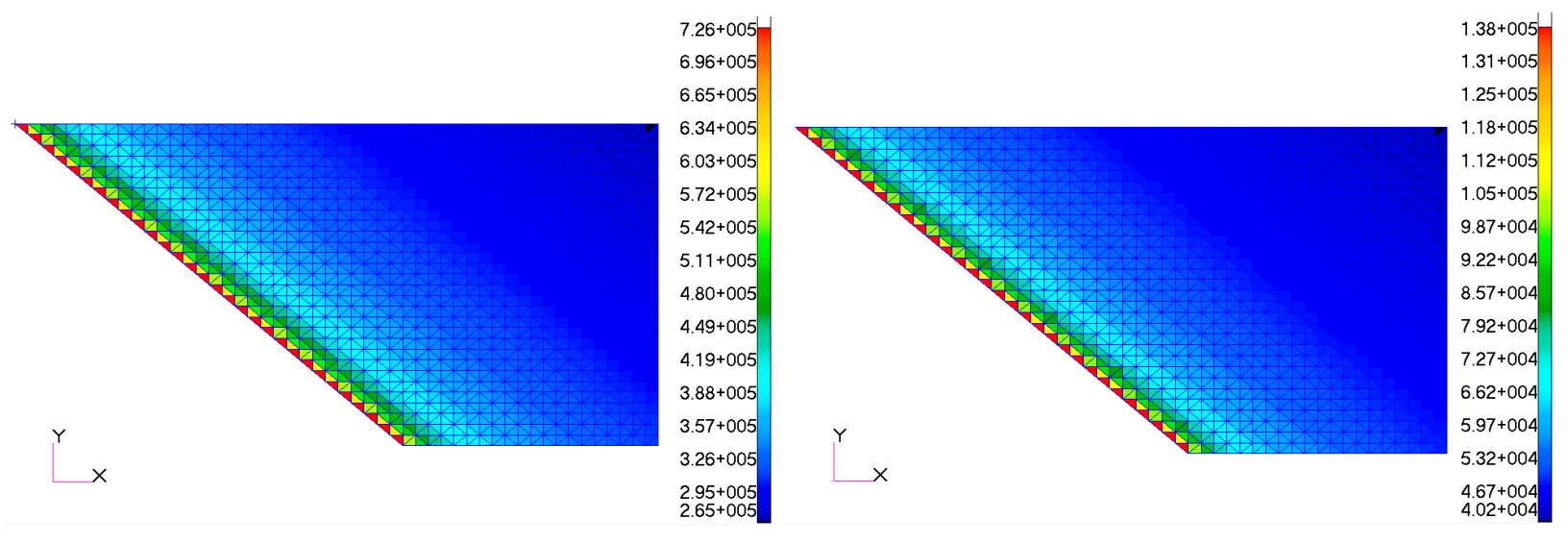

(a) Bottom surface heat flux.

(b) Top surface heat flux.

Figure 7. Aerodynamic heat flux $\left[\mathrm{W} / \mathrm{m}^{2}\right]$ on bottom and top surfaces of undeformed configuration.

to the compression on the bottom and expansion on the top resulting from the local control surface angle-ofattack. This heat flux distribution, the radiation boundary condition, and initial temperature condition were applied to the finite element model and transient thermal analysis was carried out with nodal temperatures output at selected time instants. Contour plots of the temperature distribution at $50 \mathrm{~s}$ and 2,000 s are shown in Fig. 8. Due to the heat flux being higher on the bottom surface than on the top, the bottom surface

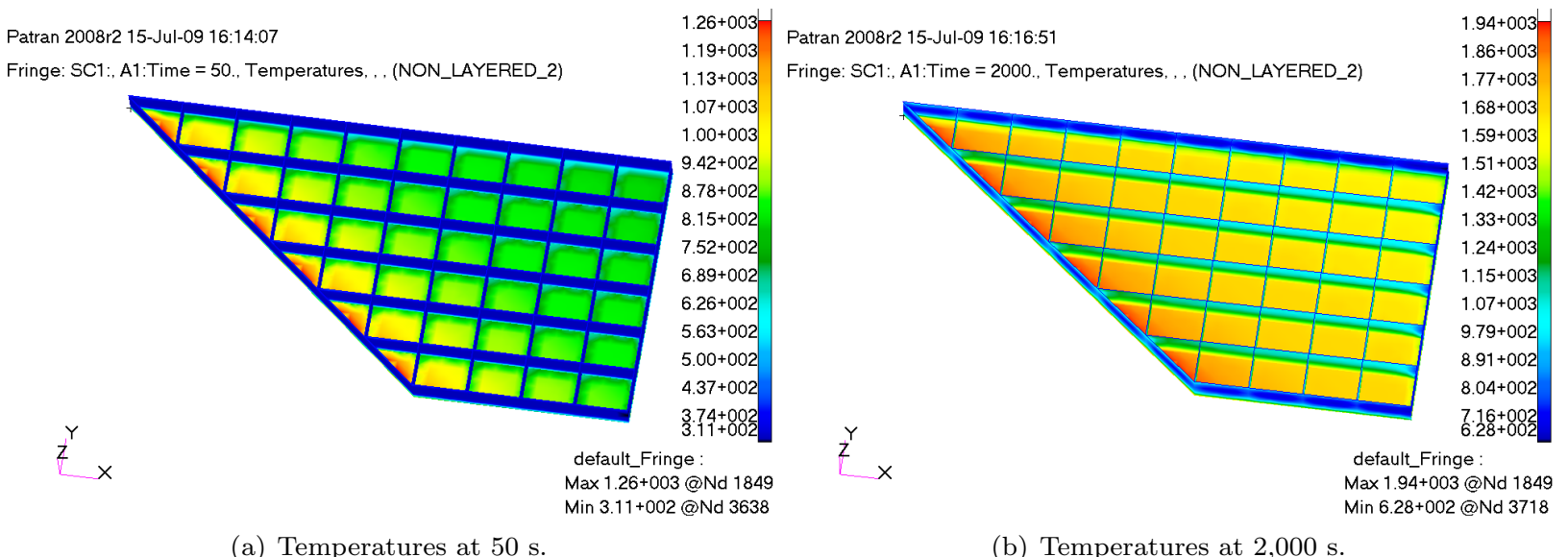

Figure 8. Nodal Temperatures $[\mathrm{K}]$ from transient thermal finite element analysis at 50 seconds and 2,000 seconds.

begins to reach its thermal equilibrium temperature much faster than the bottom surface (beginning at approximately $100-150 \mathrm{~s}$ ). The result in terms of structural deformation is that the maximum displacement increases until the bottom surface approaches the equilibrium temperature because this is the instant at which the temperature gradient between the top and bottom surfaces will be the greatest. From this point forward, the temperatures on the bottom surface will increase much slower than those on the top surface, thus decreasing the temperature gradient between the two surfaces. Thus, as the bottom surface approaches equilibrium temperature, the maximum structural displacement will begin to decrease.

Fig. 9 illustrates the above point showing that the maximum displacement at $100 \mathrm{~s}$ is greater than that at $50 \mathrm{~s}$ because the bottom surface is approaching its equilibrium temperature around this time. The maximum displacement is lower at 2,000 s than that at $100 \mathrm{~s}$ because during this interval the bottom surface remains essentially at constant temperature while the top surface continues to heat. At this point it is worth reiterating the fact that heat flux in these simulations was kept constant over time. The effect of updating the heat flux as the structure deforms on this phenomenon is unclear at this time. Further studies regarding the update of the heat flux boundary condition are thus warranted. 


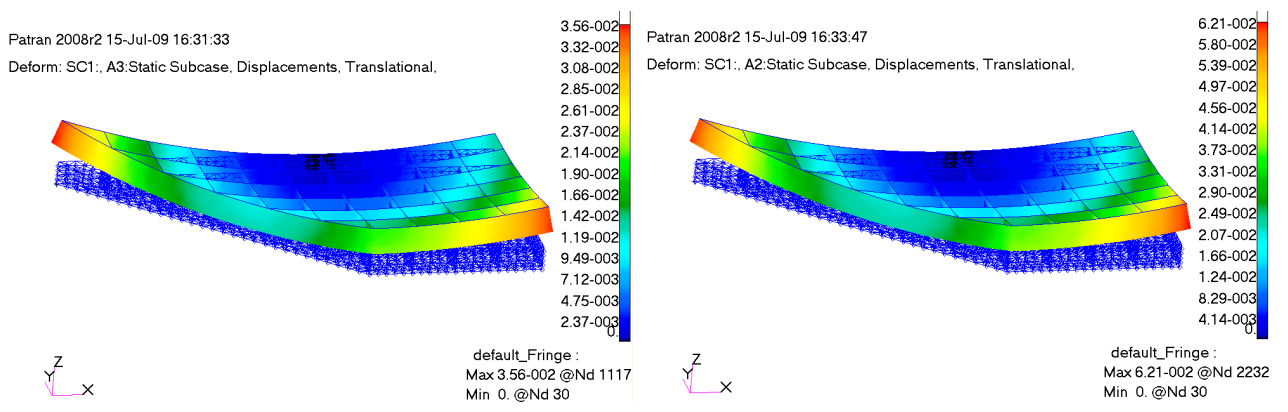

(a) Displacements at $50 \mathrm{~s}$.

(b) Displacements at $100 \mathrm{~s}$.

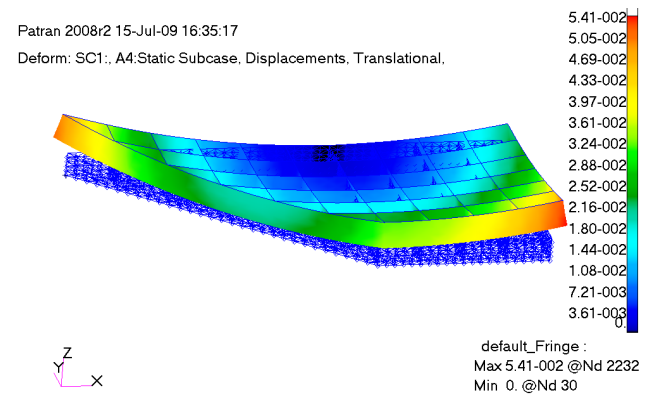

(c) Displacements at 2,000 s.

Figure 9. Structural displacements $[\mathrm{m}]$ at 50, 100, and 2,000 seconds.

With the nodal displacements known at the time instants of interest, the deformed configuration is generated at these instants and aerodynamic flow parameters are calculated at each node for each time instant. A plot of the aerodynamic pressure over the top and bottom surfaces at $100 \mathrm{~s}$ is shown in Fig. 10. The effect of angle-of-attack is seen as the pressure is higher on the bottom surface than on the top, thus generating lift. However, we see that the effect of the deformation results in the pressure decreasing along the chord on the bottom surface and increasing along the chord on the top surface. This effect is due to the fact that the curvature causes expansion of the flow over the bottom surface and compression of the flow over the top surface.

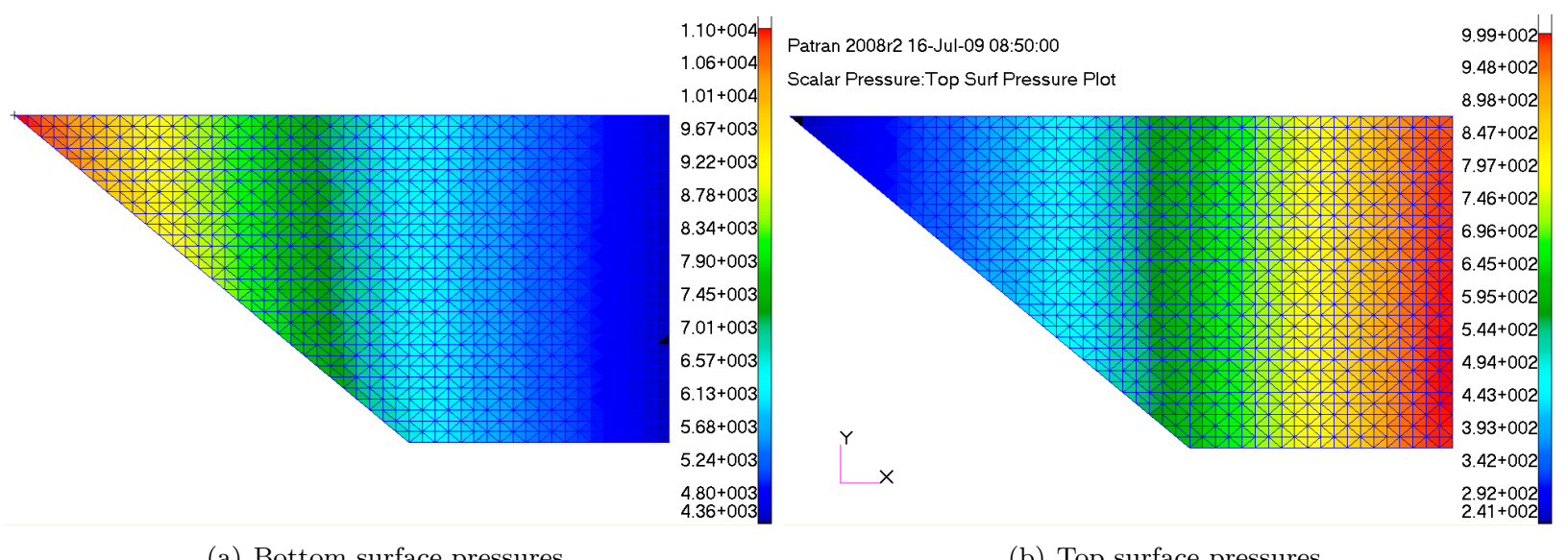

(a) Bottom surface pressures.

(b) Top surface pressures.

Figure 10. Aerodynamic pressures $[\mathrm{Pa}]$ at $100 \mathrm{~s}$.

As this study is aimed at investigating vehicle dynamics and control, it thus remains to quantify the impact of the deformations on aerodynamic forces and moments over the flight trajectory. To this end, the aerodynamic pressures were used to calculate lift and drag forces in intervals of $200 \mathrm{~s}$ out to 2,000 s. Fig. 11 shows a plot of the lift force over time for the Mach 8 cruise. We see the effect of the changing temperature gradient in the transient lift behavior. As the bottom surface approaches thermal equilibrium 
and the structure approaches its maximum displacement, the lift curve approaches its minimum value. After the bottom surface reaches equilibrium and the temperature difference between the top and bottom surfaces begins to decrease, the amount of curvature in the structure begins to decrease. This results in smaller flow turning angles, which leads to less expansion over the bottom surface and less compression over the top surface. Thus, the lift begins to increase from this point to the end of the time period considered. A similar, but opposite trend occurs for the drag behavior as shown in Fig. 12. The maximum relative change in lift was found to be $1.5 \%$, while that for drag was found to be $5.6 \%$.

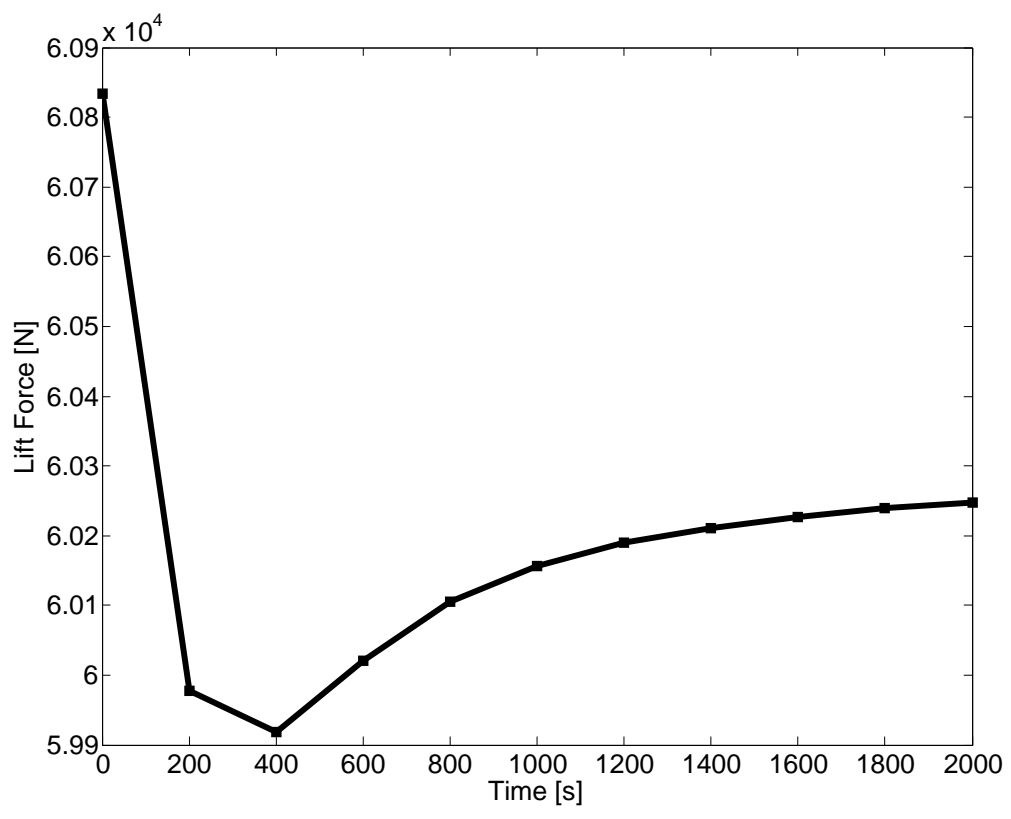

Figure 11. Lift force over time for Mach 8 cruise at $26 \mathrm{~km}$.

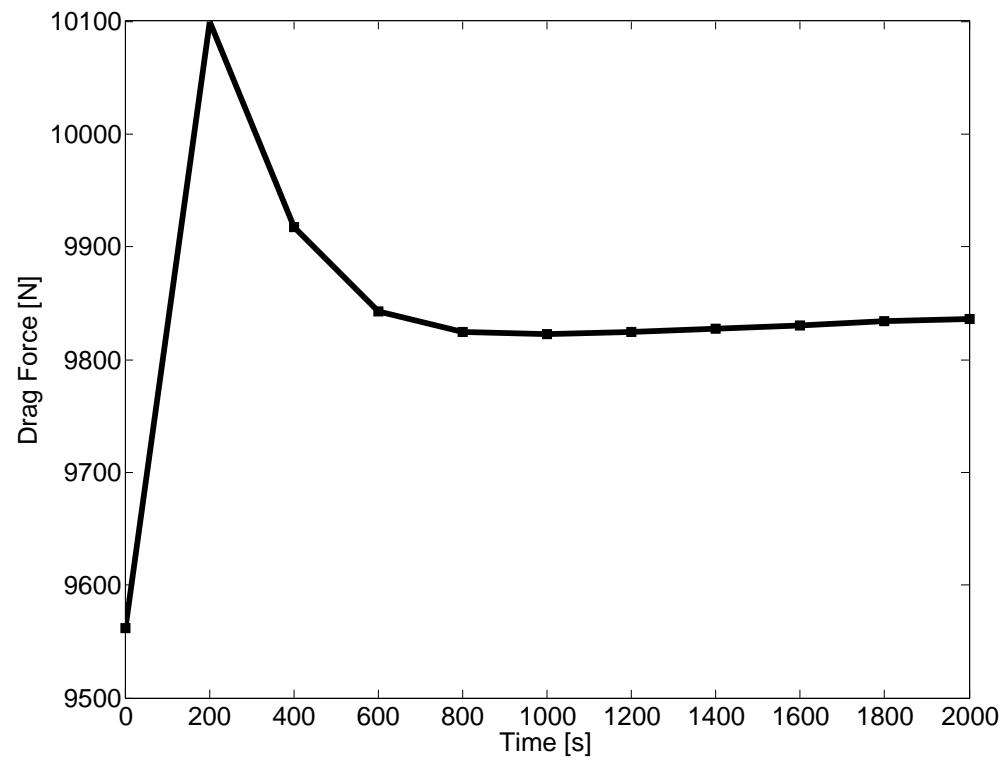

Figure 12. Drag force over time for Mach 8 cruise at $26 \mathrm{~km}$. 


\section{Control Analysis: Lift-Based Trim Routine}

As seen in Fig. 11, the structural deformation due to thermal loads will affect the lift force produced by the control surface to a certain extent. The extent to which this change in aerodynamic load affects vehicle controllability can in part be measured by the amount of control authority required to account for the changing lift force on the control surface. Based on the results presented thus far, it therefore remains to be investigated whether or not the control system will be robust to the change in aerodynamic lift resulting from heating effects. Specifically, this part of the study will investigate the extent to which control input must be applied to produce the desired lift under deformations due to aerodynamic heating. The control input to be used in this study is the angle-of-attack, $\alpha$, of the control surface. The vehicle is assumed to be at steady and level flight at initial time, and thus the targeted lift value is the amount of lift that is produced by the undeformed control surface at the initial angle-of-attack of $6^{\circ}$. The procedure to be used in this study is shown in Fig. 13.

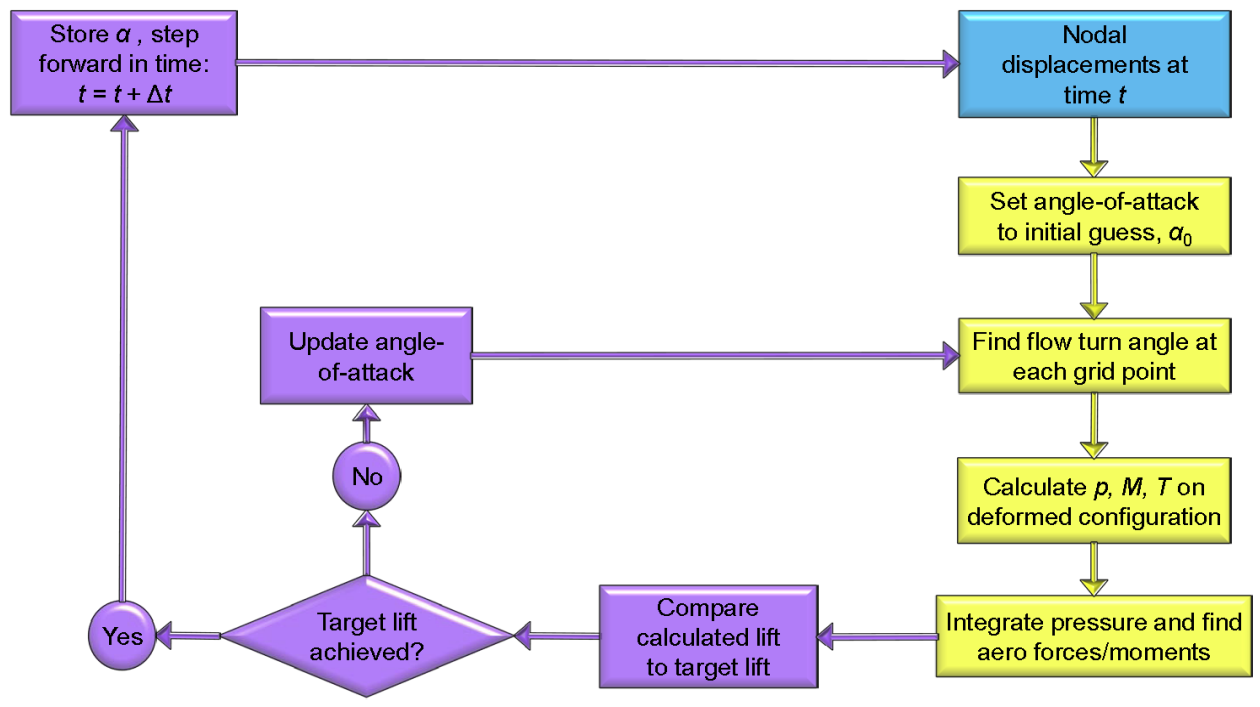

Figure 13. Procedure used in lift-based trim routine.

We begin at the first time instant by generating the corresponding deformed configuration. An initial guess value of angle-of-attack, $\alpha_{0}$, is chosen for use in the first iteration of the aerodynamic solution. The nodal flow turning angles and pressures are then found as described previously. After the pressures are integrated over the top and bottom surfaces and summed to calculate lift, the calculated lift value is compared to the target lift value to determine if the solution has converged. If not, the angle-of-attack is incremented by some amount and the aerodynamic pressures are recalculated and integrated. Iteration is continued until the calculated lift converges to the target lift. Once the target lift has been obtained, the corresponding angle-of-attack is stored and the procedure is repeated for the deformed configuration at the next time step. For this study, the Matlab routine fmincon is used for iteration and the parameter to be minimized is the absolute value of the difference between the target lift and calculated lift. This routine provides insight into the control surface actuation required to ensure that it provides constant lift over the cruise segment of the mission. As this analysis component is currently under development, results are not yet available for publication.

\section{Concluding Remarks}

An integrated aerothermoelastic modeling framework has been developed for assessment of the impact of quasi-static structural deformation on aerodynamic forces and moments as they affect vehicle controllability. The study is a step toward quantifying the changes on the control system under transient aerothermoelastic effects. As the control surfaces are expected to account for a significant portion of the aerodynamic lift, drag, and moments acting on the vehicle, this study utilized a representative control surface structure to exemplify the methodology. The thermal model consists of a finite element formulation with heat flux and radiation 
boundary conditions at the outer surfaces. The nodal values of the heat flux boundary condition were calculated using Eckert's reference temperature method with flow properties evaluated on the undeformed configuration. Transient thermal finite element analysis was carried out to generate the nodal temperatures at the time instants of interest. The temperature distributions were then used at the selected time instants to generate thermal loads on the structure and linear static structural finite element analysis was performed to calculate nodal displacements. With the nodal displacements known, the deformed configuration was generated for aerodynamic analysis. The oblique shock and Prandtl-Meyer expansion fan relations were used to calculate the aerodynamic flow parameters over the deformed control surface. Note that this study employed these relations in a local sense and used relative flow turning angles between nodes to capture the spatial variation in flow properties resulting from deformation. Integration of the calculated pressures over the top and bottom surfaces yielded the total aerodynamic lift and drag forces acting on the control surface.

The aerothermoelastic solution process was carried out at selected time instants to investigate how the lift and drag change over time for a constant-Mach, constant-altitude cruise trajectory. Though it was found that the maximum relative change in lift was less than $2 \%$ and the maximum relative change in drag was less than $6 \%$ for the trajectory points considered, the ultimate measure of the importance of this change will be its effect on vehicle control. To this end, a trim routine for characterization of the required control input under aerothermoelastic effects was developed. This routine makes use of the control surface angle-of-attack as an input parameter to modify the aerodynamic lift such that it attains a target value. The target lift value was chosen to be the lift produced in the undeformed configuration such that a constant-lift cruise trajectory is sought. Implementation of the control analysis routine and generation of results are currently ongoing at this time.

\section{Future Work}

While this study demonstrated a methodology for the inclusion of aerothermoelastic effects in an HSV flight dynamics and control simulation, further refinements will provide a more precise representation of the various phenomena considered in the analysis. For the aerothermal analysis component, relaxation of the assumption of a calorically perfect flow will more accurately capture the variation in specific heat through the boundary layer due to the high temperature. It is also necessary to include stagnation heating on the leading edge surface to more accurately characterize the heat transfer to the structure. Future work will also include the ability to update the heat flux boundary condition as the structure deforms at a specified update rate.

Though this study assessed the effect of pure thermal loading on the structure, it remains to be determined what effect aerodynamic pressure loads will have on the structural response. Independent application of aerodynamic loads will allow for a comparison between the deformation due to pressure loads and the deformation due to thermal loads and will exemplify the relative contribution of each. Furthermore, inclusion of the structural dynamics will permit analysis of the effect of oscillations about the equilibrium configuration. The inclusion of dynamics in the structural model will necessitate the use of an unsteady aerodynamic theory to calculate the flow parameters resulting from oscillations. A natural choice for such a theory would be piston theory as this method is consistent with the control-oriented modeling philosophy underlying this work.

For the control aspects of the problem, further development and implementation of the previously described trim routine is required. Once results for the quasi-static case are obtained, they can be used to guide further control and stability studies. With inclusion of the structural dynamics, more detailed analysis of the required control surface actuator dynamics can be carried out. In addition to the updates to be made to each the various analysis components, further attention will be given to the control surface model. In particular, future efforts will focus on revisiting the control surface profile, dimensions, stiffening pattern, thicknesses, materials, etc. It is expected that the control surface design will occur concurrently with the control-oriented analysis described in this paper as more is learned about the controllability and stability of the structure. The design will also be guided by the allowable temperatures, displacements, and stresses in the structure as more information regarding these parameters is ascertained. The aerothermoelastic modeling framework described in this work will not only aid in simulating the dynamics and control of hypersonic vehicles, but will also provide insight into the design of control systems, actuators, and structures for such vehicles. 


\section{Acknowledgments}

The authors acknowledge the help provided by Mr. T. Skujins (University of Michigan) with the aerodynamic solver. The authors would also like to thank Professor J. McNamara and Mr. A. Culler (The Ohio State University) for their comments regarding the aeroheating analysis. This work was supported by the Michigan-AFRL Collaborative Center in Control Science (MACCCS) under grant number FA 8650-07-2-3744 (Air Force Research Laboratory/Air Vehicles Directorate).

\section{References}

${ }^{1}$ Oppenheimer, M., Skujins, T., Doman, D., and Cesnik, C., "Canard-Elevon Interactions on a Hypersonic Vehicle," Proceedings of the 2008 AIAA Atmospheric Flight Mechanics Conference, Honolulu, Hawaii, AIAA 2008-6383.

${ }^{2}$ Skujins, T., Cesnik, C., Oppenheimer, M., and Doman, D., "Applicability of an Analytical Shock/Expansion Solution to the Elevon Control Effectiveness for a 2-D Hypersonic Vehicle Configuration," Proceedings of the 2008 AIAA Atmospheric Flight Mechanics Conference, Honolulu, Hawaii, AIAA 2008-6384.

${ }^{3}$ Oppenheimer, M. and Doman, D., "A Hypersonic Vehicle Model Developed with Piston Theory," Proceedings of the 2006 AIAA Atmospheric Flight Mechanics Conference, Hilton Head, South Carolina, AIAA 2006-6637.

${ }^{4}$ Oppenheimer, M., Skujins, T., Bolender, M., and Doman, D., "A Flexible Hypersonic Vehicle Model Developed with Piston Theory," Proceedings of the 2007 AIAA Atmospheric Flight Mechanics Conference, Hilton Head, South Carolina, AIAA 2007-6396.

${ }^{5}$ Bolender, M., Oppenheimer, M., and Doman, D., "Effects of Unsteady and Viscous Aerodynamics on the Dynamics of a Flexible Air-Breathing Hypersonic Vehicle," Proceedings of the 2007 AIAA Atmospheric Flight Mechanics Conference, Hilton Head, South Carolina, AIAA 2007-6397.

${ }^{6}$ Culler, A., Williams, T., and Bolender, M., "Aerothermal Modeling and Dynamic Analysis of a Hypersonic Vehicle," Proceedings of the 2007 AIAA Atmospheric Flight Mechanics Conference, Hilton Head, South Carolina, AIAA 2007-6395.

${ }^{7}$ Culler, A., Crowell, A., and McNamara, J., "Studies on Fluid-Structural Coupling for Aerothermoelasticity in Hypersonic Flow," Proceedings of the 50th AIAA/ASME/ASCE/AHS/ASC Structures, Structural Dynamics, and Materials Conference, Palm Springs, California, AIAA 2009-2364.

${ }^{8}$ Falkiewicz, N. and Cesnik, C., "A Reduced-Order Modeling Framework for Integrated Thermo-Elastic Analysis of Hypersonic Vehicles," Proceedings of the 50th AIAA/ASME/ASCE/AHS/ASC Structures, Structural Dynamics, and Materials Conference, Palm Springs, California, AIAA 2009-2308.

9 "Titanium Ti-6Al-2Sn-4Zr-2Mo (Ti-6-2-4-2), Duplex Annealed," http://www.matweb.com/search/DataSheet.aspx? matGUID=3a01802b122647028633c9fa10e6bae8, Last Accessed 6/30/2009.

${ }^{10}$ Myers, D., Martin, C., and Blosser, M., "Parametric Weight Comparison of Advanced Metallic, Ceramic Tile, and Ceramic Blanket Thermal Protection Systems," Tech. Rep. TM 2000-210289, NASA, June 2000.

${ }^{11}$ Holman, J., Heat Transfer, McGraw-Hill, Inc., 8th ed., 1997.

${ }^{12}$ Eckert, E., "Engineering Relations for Heat Transfer and Friction in High-Velocity Laminar and Turbulent BoundaryLayer Flow Over Surfaces with Constant Pressure and Temperature," Transactions of the American Society of Mechanical Engineers, Vol. 78, No. 6, August 1956.

${ }^{13}$ Eckert, E., "Survey on Heat Transfer at High Speeds," Technical Report ARL 189, Aeronautical Research Laboratory, University of Minnesota, Minneapolis, MN, December 1961.

${ }^{14}$ Huebner, K., Dewhirst, D., Smith, D., and Byrom, T., The Finite Element Method for Engineers, John Wiley and Sons, Inc., 4th ed., 2001.

${ }^{15}$ Gould, P., Introduction to Linear Elasticity, Springer-Verlag, 2nd ed., 1994.

${ }^{16}$ Chandrupatla, T. and Belegundu, A., Introduction to Finite Elements in Engineering, Prentice-Hall, Inc., 3rd ed., 2002.

17 "Equations, Tables, and Charts for Compressible Flow," Tech. Rep. NACA-1135, National Advisory Committee for Aeronautics, Ames Aeronautical Laboratory, Moffett Field, CA, 1953.

${ }^{18}$ Anderson, J., Fundamentals of Aerodynamics, McGraw-Hill, Inc., 3rd ed., 2001. 